\title{
CRISPR-CaS SYSTEMS: A POTENTIAL TOOL TO REDUCE THE GLOBAL BURDEN OF ANTIMICROBIAL RESISTANCE
}

\author{
Yusuf Amuda Tajudeen ${ }^{1 *}$, Iyiola Olatunji Oladunjoye ${ }^{1,2}$, Habiba Iliyasu Atta ${ }^{3}$, Kehinde Eyitayo Oyediji ${ }^{1}$ \\ Address (es): \\ ${ }^{1}$ University of Ilorin, Faculty of Life Sciences, Department of Microbiology, 45 Edun Street, 240251, Ilorin, Nigeria, +2347062063691. \\ ${ }^{2}$ Group Executive (Health, Agriculture, \& Life Sciences), Rouleaux Foundation, Lagos, Nigeria. \\ ${ }^{3}$ Department of Microbiology, Faculty of Life Sciences, Ahmadu Bello University, Zaria, Nigeria.
}

*Corresponding author: tajudeenamudayusuf@gmail.com

ABSTRACT

https://doi.org/10.36547/be.273

\section{ABSTRACT}

Antimicrobial resistance is one of the top global health threats driving our planet to the post-antibiotic era. The formulation of antibiotics is expensive and timeconsuming, and this may have delayed the production of new ones. Therefore, approaches such as Clustered Regularly Interspersed Short Palindromic Repeatsassociated protein-Cas-CRISPR-Cas, a system which is capable of reverting Antimicrobial Resistance Genes (ARGs) could be the potential means of significantly reducing the burden of antimicrobial resistance globally. In this perspective, we highlight how this system has been previously applied in vivo and in vitro studies to tackle antibiotics resistance, stating its relevance as well as challenges while providing recommendation for the effective use of this system.

Keywords: CRISPR-Cas, Antimicrobial Resistance, Antibiotics

\section{INTRODUCTION}

Antimicrobial resistance (AMR) occurs when microorganisms including bacteria, viruses, fungi, and parasites, no longer respond to the medications (antibiotics, antivirals, antifungals, and antiparasitics) that previously inhibited their growth effectively or destroy them (O Neil, 2014). Internationally, AMR is a growing public health threats which is currently estimated by World Health Organization (WHO) to cause $>700,000$ deaths per annum worldwide. And by 2050, this figure may substantially rise to 10 million with a global economy cost of approximately US\$100 trillion per annum if adequate measures are not put in place to salvage the situation, it could result in an issue of public health concern (O Neil, 2014). Several factors are known to contribute to AMR, one of which is the excessive use and misuse of antimicrobial drugs, particularly those used for treatment of bacterial infections. This has led to the development of resistance to antibiotics with some of the currently available medications (Goossens et al, 2005). Over the years, pharmaceutical companies have been trying to screen, test and develop new antibiotics in a bid to control AMR, however, the biggest challenge to this approach is inadequate funding. This has led to a decrease in production of new antibiotics expected to be active against resistant bacterial strains, whereas the previously discovered ones are being overly used, contributing to an increased resistance in pathogenic bacteria (Benjamin, 2020). Consequently, some pharmaceutical companies that managed to discover new antibiotics still go bankrupt sometimes, and this is evident from American antibiotic start-ups like Achaogen and Aradigm (New York Tine, 2019). It is also pertinent to note that out of the new antibiotics that enter the clinical trials only considerably small percentages usually got approval from the Food and Drug Administration (FDA), thus, making it difficult to combat the new emerging resistant strains (Pew Charitable Trusts, 2019). Evidently, at the end of 2019, out of the forty-one antibiotics in the clinical trials, only four $(\sim 10 \%)$ had been approved - this is an indication that the acceptance rate for newly discovered antibiotics is considerably low (Pew Charitable Trusts, 2019). With regards to this problem, a nucleic acid-based antibacterial treatment should be exploited to treat such infections and also to counteract resistance from multidrug resistant bacteria.

Table 1 Various antimicrobial studies that used CRISPR as a gene editing tool. (Citorik et al, 2014; Bilkard $\boldsymbol{e t}$ al, 2014; Yosef $\boldsymbol{e t}$ al, 2015 \& Kim et al, 2016)

\begin{tabular}{llc}
\hline S/N & Antimicrobial studies & \multicolumn{1}{c}{ Authors and Year } \\
\hline 1. & $\begin{array}{l}\text { Sequence-specific antimicrobials using efficiently delivered } \\
\text { RNA-guided nucleases. }\end{array}$ & Citorik RJ, Mimee M, Lu TK. Nat Biotechnol 2014;32: \\
\hline 2. & $\begin{array}{l}\text { Exploiting CRISPR-Cas nucleases to produce sequence } \\
\text { specific antimicrobials. }\end{array}$ & $\begin{array}{l}\text { Bikard D, Euler CW, Jiang W, Nussenzweig PM, Goldberg } \\
\text { GW, Duportet X, et al. Nat Biotechnol 2014;32: 1146-1150 }\end{array}$ \\
\hline 3. & $\begin{array}{l}\text { Temperate and lytic bacteriophages programmed to sensitize } \\
\text { and kill antibiotic-resistant bacteria. }\end{array}$ & $\begin{array}{l}\text { Yosef I, Manor M, Kiro R, Qimron U. PNAS 2015; 112(23): } \\
\text { 7267-7272. }\end{array}$ \\
\hline 4. & $\begin{array}{l}\text { CRISPR/Cas9-Mediated Re-Sensitization of Antibiotics- } \\
\text { Resistant Escherichia-coli Harboring Extended-Spectrum } \beta- \\
\text { Lactamases. }\end{array}$ & $\begin{array}{c}\text { Kim JS, Cho DH, Park M, Chung WJ, Shin D, Ko KS, et al. } \\
\text { J Microbiol Biotechnol 2016; 26(2): 394-401. }\end{array}$ \\
\hline
\end{tabular}

CRISPR-Cas is a gene editing tool whose operation is based on adaptive immune mechanisms, and it is commonly used by bacteria and archaea as a preventive measure against invasive pathogens like viruses, and the phage genome (de la Fuente-Nunez et al, 2017). The CRISPR-Cas system is composed of Cas9-a DNA cutting endonuclease enzyme that works like molecular scissors, a guide RNA (gRNA) which direct the Cas9 to the targeted sequence in the genome that needs to be cleaved (Cui $\boldsymbol{e t} \boldsymbol{a l}$, 2017). For instance, when phage virus attack bacteria like Escherichia coli, it introduces its DNA sequence (Adenine, Thymine, Cytosine, and Guanine) into the bacteria cell, and forcibly seize the cellular machinery of the infected bacteria to replicate more copies of itself to initiate infection. However, E.coli counters the invading phages using its gRNA to bind to the targeted DNA sequence of the phages, while Cas9 enzyme alongside with invading phages component called PAM (Protospacer Adjacent Motif) finally makes a precise cleave on both strands of the double helix DNA of the viruses into two segments, thus, disabling the virus (Cui $\boldsymbol{e t}$ al, 2017). One of these cut segments is inserted into specific area of E.coli genome to build immune memories of the phage invaders by copying the phage nucleotide sequence into its RNA i.e. gRNA. Upon subsequent attack, the endonuclease enzyme Cas9 attached to the gRNA will recognize the pieces of phages RNA inside E.coli that is the same with the nucleotide sequence from the gRNA, and with Cas9 enzyme, the DNA of invading phages will be precisely cleaved and disabled (Bilkard et al, 2014; Cui et al, 2017). This system is also used in pathogenic bacteria like Campylobacter jejuni, Streptococcus pyogenes, and Staphylococcus aureus, and can therefore be exploited from these organisms, and through its modification for use in human, can be a potential means to combat antibiotic-resistant bacteria. And may possibly be the expected cure for other diseases of genetic origin like sickle cell anaemia, cancer, cystic fibrosis, molecular dystrophy, blood disorder, and viral infections like AIDS and SARSCoV-2 (Clara, 2019; Cui et al, 2017 \& de la Fuente-Nunez et al, 2017) 


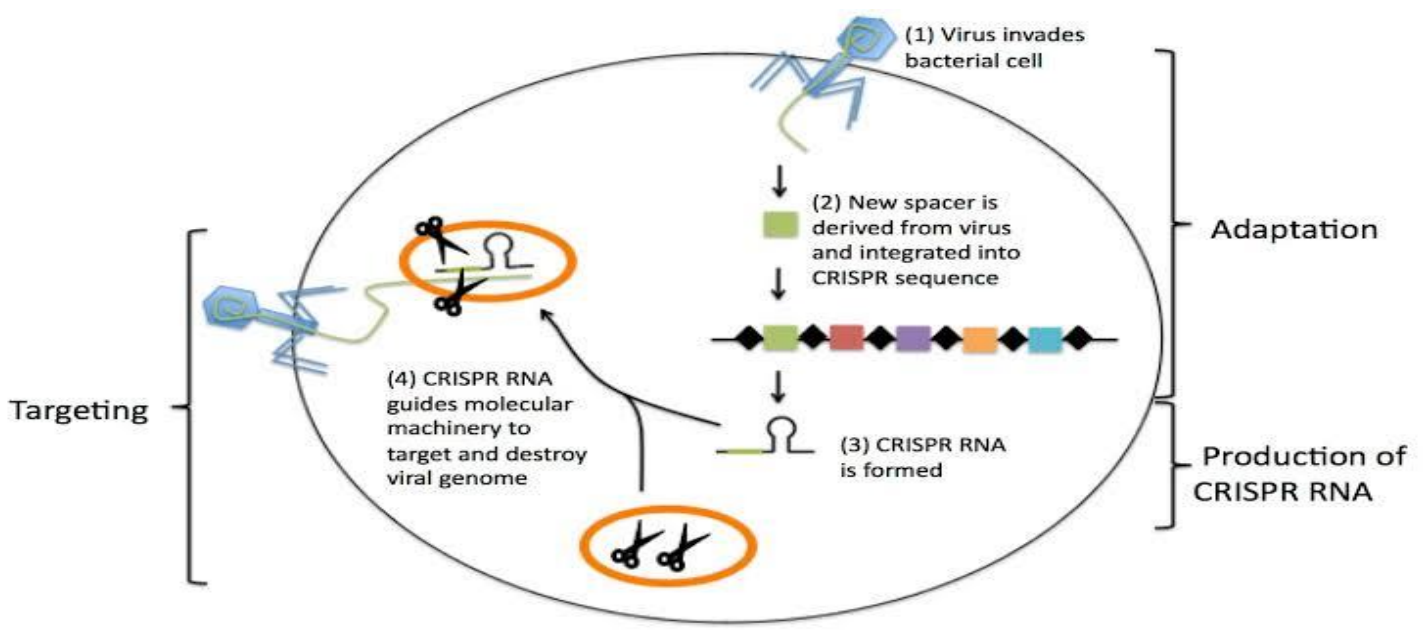

Figure 1 Mechanism of action of CRISPR-Cas as a gene editing tool in bacteria to counter the invading virus (Barrangou et al, 2014)

According to the research by Law BJC and colleagues, a newly developed CRISPR-Cas9 pro-active genetic system could be used as a gene editing tool to eliminate virulence factors such as resistance-encoding genes on bacteria plasmid as well as resistance determinants in commensal bacteria species (Law et al, 2018). Through this system, the antibiotics-resistance genes (ARGs) known to confer resistance to antibiotics can be targeted by an already designed CRISPR guide RNA which will induce a snip into the double stranded DNA (dsDNA) of the antibiotics resistant bacteria using the Cas9 endonuclease enzyme (already coded with a targeted sequence that enhances the cytotoxicity of antibiotic resistant cells), and then mutate/revert the ARGs into antibiotics sensitive ones, thereby rendering the antibiotics more effective, and eliminate the possibility of resistance by resistant bacteria (Cui $\boldsymbol{e t}$ al, 2016). In a review by de la FuenteNunez et al, (2017) this approach operates effectively in vitro by successfully eliminating the targeted pathogenic bacteria carrying the ARGs. In another study by Bikard $\boldsymbol{e t}$ al, (2014) it also works in vivo using an invertebrate mouse skin model colonized with Staphylococcus aureus.

However, adopting this approach in humans is not without its challenges, some of which include non-specific targeting/missing of the targeted genome and making unwanted edits associated with Cas9, thereby leading to elimination of the pathogenic microbes along with the commensal bacterial species, and this can lead to microbial imbalance, thus exposing humans to several infectious diseases, although, this can be corrected by administering CRISPR-Cas treatment together with probiotics for selective elimination of the resistant strains, while leaving the commensal bacterial species (Aslam et al, 2020) Another challenge with this approach is that there are also safety and ethical concerns on the use of gene editing tool on human subjects.

\section{CONCLUSION}

In conclusion, the discovery of CRISPR-Cas system has evolved considerably as an important tool in genomic engineering for gene editing, deletion, and tweaking, to proffer cure for diseases of genetic origin as well as stimulate genetic traits. However, using this system as a tool for delivering antimicrobials is still in its infancy and has gained less attention from researchers despite its efficacy in tackling the targeted antibiotic resistant bacteria. It is therefore recommended that attention should be paid towards the use of this system as an antimicrobial tool. Further studies should focus on the safety of the use of this system in humans, serving as a potential means to reduce the global health burden of antimicrobial resistance.

Conflict of interest: The authors declare no conflict of interest.

\section{REFERENCES}

Aslam, B., Rasool, M., Idris, A., Muzammil, S., Alvi, F.R., Khurshid, M., Rasool, H.M., Zhang, D., Ma, Z., \& Baloch, Z. (2020). CRISPR-Cas system: a potential alternative tool to cope antimicrobial resistance. Antimicrobial Resistance and Infection Control, 9: 131. https://doi.org/10.1186/s13756-020-00795-6.

Barrangou, R., \& Murraffini L. (2020). CRISPR-Cas Systems: Prokaryotes Upgrade to Adaptive Immunity. Molecular Cell, 54: 234-244.

Benjamin P. (2020). Despite an overwhelming global need for pharmaceutical companies to develop more antibiotics, there's little financial incentive to encourage them to act. Nature- Antimicrobial Resistance Outlook, 586: S50-S52. https://doi.org/10.1038/d41586-020-02884-3.

Bikard, D., Euler, C.W., Jiang, W., Nussenzweig, P.M., Goldberg, G.W., Duportet, X., Fischetti, A.V., \& Luciano M.A. (2014). Exploiting CRISPR-Cas nucleases to produce sequence-specific antimicrobials. Nature Biotechnology, 32:1146-1150. https://doi.org/10.1038/nbt.3043.

Citorik, R.J., Mimee, M., \& Lu T.K. (2014). Sequence-specific antimicrobials using efficiently delivered RNA-guided nucleases. Nature Biotechnology, 32: 1141-1145.

Clara, R.F. (2019). 7 Diseases CRISPR Technology Could Cure, CRISPR technology offers the promise to cure any human genetic disease. Which are the candidates to be the first one? https://www.labiotech.eu/crispr/crispr-technology-cure-disease/.

Cui, L., \& Bikard D. (2016). Consequences of Cas9 cleavage in the chromosome of Escherichia coli. Nucleic Acids Research, 44(9): 4243-51. https://doi.org/10.1093/nar/gkw223

de la Fuente-Nunez, C., Torres, M.D., Mojica, F.J., \& Lu TK. (2017). Next-generation precision antimicrobials: towards personalized treatment of infectious diseases. $\begin{array}{llll}\text { Current Opinion in } & \text { 95-102. }\end{array}$ https://doi.org/10.1016/j.mib.2017.05.014.

Goossens, H., Ferech, M., Vander, R., \& Elseviers M. (2005). Outpatient antibiotic use in Europe and association with resistance: a cross-national database study. The Lancet, 365: 579-587. https://doi.org/10.1016/S0140-6736(05)17907-0.

Kim, J.S., Cho, D.H., Park, M., Chung, W.J., Shin, D., Ko, K.S., \& Kwon D.H. (2016). CRISPR/Cas9-Mediated Re-Sensitization of Antibiotics-Resistant Escherichia-coli Harboring Extended-Spectrum $\beta$-Lactamases. Journal of Microbiology and Biotechnology, 26(2): 394-401. https://doi.org/10.4014/jmb.1508.08080.

Law, B.J.C., Zhuo, Y., Winn, M., Francis, D., Zhang, Y.X., Samborskyy, M., Murphy, A., Ren, L., Leadlay, F.P., \& Micklefield J. (2018). A vitamin K-dependent carboxylase orthologue is involved in antibiotic biosynthesis. Nature Catalysis, 1(12):977-84. https://doi.org/10.1038/s41929-018-0178-2.

O'Neil J. (2014). Antimicrobial Resistance: Tackling a crisis for the health and wealth of nations. https://amr-review.org.

Pew Charitable Trusts. (2019). Tracking the Global Pipeline of Antibiotics in Development. https://www.pewtrusts.org/research-and-analysis/issuebriefs/2019/09/tracking-the-global-pipeline-of-antibiotics-in-development.

The New York Times. (2019). Crisis Looms in Antibiotics as Drug Makers Go Bankrupt. https://www.nytimes.com/2019/12/25/health/antibiotics-newresistance.html.

Yosef, I., Manor, M., Kiro, R.,\& Qimron U. (2015). Temperate and lytic bacteriophages programmed to sensitize and kill antibiotic-resistant bacteria. PNAS, 112(23): 7267-7272. https://doi.org/10.1073/pnas.1500107112 